\title{
Magnetic-Field-Induced Quantum Phase Transitions in a van der Waals Magnet
}

\author{
Siwen Li, ${ }^{1}$ Zhipeng Ye, ${ }^{2}$ Xiangpeng Luo, ${ }^{1}$ Gaihua Ye, ${ }^{2}$ Hyun Ho Kim, ${ }^{3}$ Bowen Yang, ${ }^{3}$ Shangjie Tian, ${ }^{4}$ \\ Chenghe Li, ${ }^{4}$ Hechang Lei, ${ }^{4}$ Adam W. Tsen, ${ }^{3}$ Kai Sun, ${ }^{1}$ Rui He®, ${ }^{2, *}$ and Liuyan Zhao ${ }^{1, \dagger}$ \\ ${ }^{1}$ Department of Physics, University of Michigan, 450 Church Street, Ann Arbor, Michigan 48109, USA \\ ${ }^{2}$ Department of Electrical and Computer Engineering, Texas Tech University, \\ 910 Boston Avenue, Lubbock, Texas 79409, USA \\ ${ }^{3}$ Institute for Quantum Computing, Department of Chemistry, and Department of Physics and Astronomy, \\ University of Waterloo, Waterloo, 200 University Avenue W, Ontario N2L 3G1, Canada \\ ${ }^{4}$ Department of Physics and Beijing Key Laboratory of Opto-electronic Functional Materials \& \\ Micro-nano Devices, Remin University of China, Beijing 100872, China
}

(Received 17 January 2020; revised manuscript received 24 February 2020; accepted 27 February 2020; published 31 March 2020)

\begin{abstract}
Exploring new parameter regimes to realize and control novel phases of matter has been a main theme in modern condensed matter physics research. The recent discovery of two-dimensional (2D) magnetism in nearly freestanding monolayer atomic crystals has already led to observations of a number of novel magnetic phenomena absent in bulk counterparts. Such intricate interplays between magnetism and crystalline structures provide ample opportunities for exploring quantum phase transitions in this new $2 \mathrm{D}$ parameter regime. Here, using magnetic field- and temperature-dependent circularly polarized Raman spectroscopy of phonons and magnons, we map out the phase diagram of chromium triiodide $\left(\mathrm{CrI}_{3}\right)$ that has been known to be a layered antiferromagnet (AFM) in its 2D films and a ferromagnet (FM) in its threedimensional (3D) bulk. However, we reveal a novel mixed state of layered AFM and FM in 3D $\mathrm{CrI}_{3}$ bulk crystals where the layered AFM survives in the surface layers, and the FM appears in deeper bulk layers. We then show that the surface-layered AFM transits into the FM at a critical magnetic field of $2 \mathrm{~T}$, similar to what was found in the few-layer case. Interestingly, concurrent with this magnetic phase transition, we discover a first-order structural phase transition that alters the crystallographic point group from $C_{3 i}$ (rhombohedral) to $C_{2 h}$ (monoclinic). Our result not only unveils the complex single-magnon behavior in 3D $\mathrm{CrI}_{3}$, but it also settles the puzzle of how $\mathrm{CrI}_{3}$ transits from a bulk FM to a thin-layered AFM semiconductor, despite recent efforts in understanding the origin of layered $\mathrm{AFM}$ in $\mathrm{CrI}_{3}$ thin layers, and reveals the intimate relationship between the layered AFM-to-FM and the crystalline rhombohedral-tomonoclinic phase transitions. These findings further open opportunities for future $2 \mathrm{D}$ magnet-based magnetomechanical devices.
\end{abstract}

DOI: 10.1103/PhysRevX.10.011075

Subject Areas: Condensed Matter Physics

\section{INTRODUCTION}

Chromium triiodide $\left(\mathrm{CrI}_{3}\right)$ stands out among the large family of van der Waals (VDW) ferromagnets (FMs) investigated so far, because its isolated atomic crystal is one of the first two to realize two-dimensional (2D) ferromagnetic long-range order [1,2] in the monolayer limit, and more importantly, it is the first-ever interlayer

\footnotetext{
* Corresponding author.

rui.he@ttu.edu

Corresponding author. lyzhao@umich.edu

Published by the American Physical Society under the terms of the Creative Commons Attribution 4.0 International license. Further distribution of this work must maintain attribution to the author(s) and the published article's title, journal citation, and DOI.
}

antiferromagnetic (AFM) semiconductor [3-6] in its multilayer form. This phenomenon also makes $\mathrm{CrI}_{3}$ distinct from the 2D intralayer AFMs [7-9]. From an application perspective, such unique magnetic properties of $\mathrm{CrI}_{3}$ have already drawn enormous interest in developing novel device functionalities that are tunable by either magnetic [3-6] or electric [10-12] fields. From a fundamental science point of view, extensive static and dynamic measurements in both three-dimensional (3D) bulk $[13,14]$ and 2D film $[3-6,15,16] \mathrm{CrI}_{3}$ have been performed to understand the mechanism of the magnetism that has remained elusive until now. Our study here approaches the nature of $\mathrm{CrI}_{3}$ magnetism through exploring the magnetic field dependence of spin-wave excitations in $\mathrm{CrI}_{3}$ and interrogating the interplay between the magnetic order and the crystalline structure.

In its bulk form, $\mathrm{CrI}_{3}$ goes through a monoclinic $\left(C_{2 h}\right)$ to rhombohedral $\left(C_{3 i}\right)$ structural phase transition at 
$T_{s}=220 \mathrm{~K}$ and develops a FM long-range order at $T_{\mathrm{FM}}=$ $61 \mathrm{~K}$ [13]. Across $T_{s}$, the major structural change involves the shearing of the VDW bonded $\mathrm{CrI}_{3}$ layers of the honeycomb lattice from a tilted to an aligned $\mathrm{ABC}$ stacking sequence [13]. Below $T_{\mathrm{FM}}$, all the spin moments within and between layers align along the out-of-plane direction [13], and a spin-wave gap of approximately $1 \mathrm{meV}$ emerges at the Brillouin-zone-center $\Gamma$ point as a result of the Ising-type exchange anisotropy [14]. The magnetic and crystallographic degrees of freedom couple strongly in bulk $\mathrm{CrI}_{3}$ as evidenced by the enhanced reduction of interlayer spacing below $T_{\mathrm{FM}}$ [13]. In its thin-layer form, the $\mathrm{CrI}_{3}$ magnetism is known to survive below $T_{\mathrm{AFM}}=45 \mathrm{~K}$; however, in a unique layered $\mathrm{AFM}$ order in which spins align along the same out-of-plane direction within each layer and alternate to the opposite orientation between adjacent layers [3-6], while its crystal structure is much less studied experimentally. The origin of this crossover from the FM in bulk to the layered AFM in thin films, as well as the role of the crystallographic structure in this transition, remains as outstanding open question in the field of 2D magnetism.

\section{EXPERIMENT AND ANALYSIS}

In order to probe both the magnetic and crystallographic degrees of freedom in $\mathrm{CrI}_{3}$, we carry out polarized Raman spectroscopy to detect the symmetry-resolved collective excitations of spin precessions (i.e., magnons) [17] and lattice vibrations (i.e., phonons) [18-21], respectively. To further reveal the interplay between these 2 degrees of freedom, we perform both temperature- and magnetic fielddependent Raman measurements covering a temperature $(T)$ range from room temperature down to $10 \mathrm{~K}$ and a magnetic field $(B)$ range from 0 up to $7 \mathrm{~T}$. Because the stray magnetic fields cause the Faraday rotation of linearly polarized light transmitted through the objective in close proximity to the magnet [22], we choose circularly polarized light to perform reliable selection rule measurements (see the Supplemental Material Sec. 1 [23] for Raman selection rules in the circular polarization basis for $\mathrm{CrI}_{3}$, and see Methods for details of the Raman measurements).

\section{A. Magnetism-related Raman modes}

We start by showing Raman spectra in both parallel and crossed circularly polarized channels labeled as LL and LR in Fig. 1(a), respectively, taken on a freshly cleaved $3 \mathrm{D} \mathrm{CrI}_{3}$ crystal at $T=10 \mathrm{~K}$ and $B=0 \mathrm{~T}$, where $\mathrm{LL}(\mathrm{R})$ stands for incident and scattered light being left and left (right) circularly polarized, respectively. We categorize all the observable Raman modes into three categories based on their symmetry properties. The first category contains phonon modes of either $A_{g}$ or $E_{g}$ symmetry of the $C_{3 i}$ point group as reported before using linearly polarized light $[15,24,25]$. Under the circular polarization basis in Fig. 1(a), the $A_{g}$ phonon modes [labeled $P\left(A_{g}\right)$ ] appear only in the LL channel, and the $E_{g}$ ones $\left[P\left(E_{g}\right)\right]$ are solely in the LR channel, as expected for the rhombohedral crystal structure. The second group consists of two modes $M_{1}$ and $M_{2}$ that were previously shown to be antisymmetric and attributed to surface magnetic excitations [15], showing up in the LL channel here. The third kind is exclusively very lowfrequency modes $M_{0}$ that, however, have neither been detected experimentally $[15,24,25]$ nor predicted theoretically $[26,27]$ before in Raman studies and are present in both LL and LR channels. Symmetrywise, these $M_{0}$-type modes violate the selection rules for the rhombohedral crystal structure and the FM order. Energywise, their frequencies of approximately $4 \mathrm{~cm}^{-1}(0.49 \mathrm{meV})$ are close to the reported bulk spin-wave gap, which is on the order of $1 \mathrm{meV}$ [14]. We note that the $M_{0}$ intensity is stronger in the anti-Stokes than the Stokes channel, possibly due to the resonance excitation to the charge-transfer transition in $\mathrm{CrI}_{3}$ and the broken time-reversal symmetry of $M_{0}$.

To explore the nature of these potentially magnetismrelated Raman modes $M_{0-2}$ and investigate the magnetoelastic coupling between magnetic and phononic modes, we carefully carry out out-of-plane magnetic field- $(B \perp a b)$ dependent Raman measurements at $10 \mathrm{~K}$ and show the results in the LL channel in Fig. 1(b) (see Supplemental Material Sec. 2 [23] for extended data of magnetic fielddependent Raman spectra on $3 \mathrm{D} \mathrm{CrI}_{3}$ bulk). First of all, it is clear that the frequencies of $M_{0}$ scale linearly with the magnetic field, consistent with what would be expected for spin-wave excitations, despite a discontinuity at approximately $2 \mathrm{~T}$ and a much more complex pattern for the magnetic fields lower than $2 \mathrm{~T}$. In addition, at $0 \mathrm{~T}, M_{0}$-type modes soften toward $0 \mathrm{~cm}^{-1}$ when approaching the critical temperature $T_{c}$ of $45 \mathrm{~K}$ from below, which is consistent with the expected trend of $\sqrt{T_{c}-T}$ for an order parameter under the mean-field theory [28,29] [Fig. 1(d) top panel]. Based on both the linear magnetic field dependence and the softening of $M_{0}$ frequencies, we confidently assign $M_{0}$-type modes to be spin-wave excitations. Second, the $M_{1}$ and $M_{2}$ show no magnetic field dependence at all below $2 \mathrm{~T}$ and disappear at higher magnetic fields. Although their antisymmetric selection rule is indicative of their magnetic origin [17] and their temperature-dependent intensities mimic closely that of the magnetic order parameter [28,29] [Fig. 1(d) middle and bottom panels], such a magnetic field independence of $M_{1}$ and $M_{2}$ before $2 \mathrm{~T}$ immediately rules out the possibility of them being conventional spin-wave excitations. More insights about their potential nature are discussed later in Fig. 2. Third, the phonons exhibit contrasting selection rules below and above $2 \mathrm{~T}$ while their frequencies remain more or less independent of magnetic field. For example, the $E_{g}$ mode (at approximately $109 \mathrm{~cm}^{-1}$ ) leaks into the LL channel [Fig. 1(b)], and the $A_{g}$ modes (at approximately 79 and $129 \mathrm{~cm}^{-1}$ ) show up in the LR channel above $2 \mathrm{~T}$ (see Supplemental Material Sec. 2 [23]). 

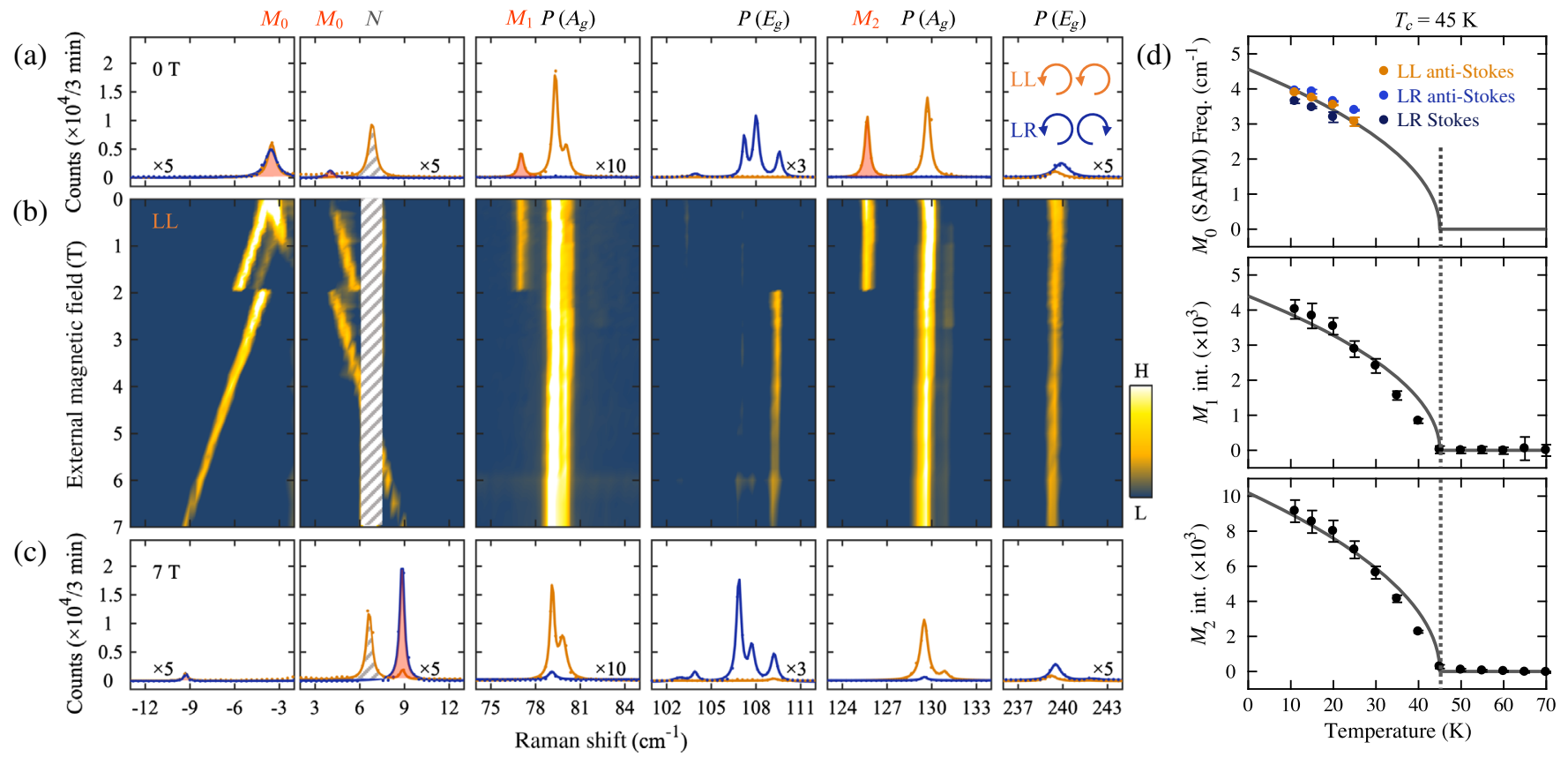

FIG. 1. Identifying the magnetism-related Raman modes in $3 \mathrm{D} \mathrm{CrI}_{3}$. (a) Raman spectra taken at $10 \mathrm{~K}$ and $0 \mathrm{~T}$ in the LL and LR channels. LL(R) stands for the polarization channel in which the incident and scattered light is left and left (right) circularly polarized, respectively. Solid dots are raw data points and solid lines are Lorentzian fits. The multiplication factors to the spectra intensities are labeled in the corresponding panels. The Raman modes are labeled as $M_{0,1,2}$ for the magnetism-related ones (shaded in orange), $P\left(A_{g}\right)$ and $P\left(E_{g}\right)$ for the phonon modes of the $A_{g}$ and $E_{g}$ symmetries of the $C_{3 i}$ point group, respectively, and $N$ for the noise line from the incident light (shaded in gray stripes). Inset shows the legends for the polarization channels. (b) A color map of magnetic field-dependent Raman spectra taken over a magnetic field range of 0-7 T at $10 \mathrm{~K}$ in the LL channel. The gray stripe-patterned shade is to block the noise line. (c) Raman spectra taken at $10 \mathrm{~K}$ and $7 \mathrm{~T}$ in the LL and LR channels. (d) Temperature dependence of the $M_{0}$ frequency (including data for the LL anti-Stokes, LR anti-Stokes, and Stokes shifts) and $M_{1,2}$ intensities at zero magnetic field ( $B=0 \mathrm{~T}$ ) showing a clear onset at $T_{c}$ of $45 \mathrm{~K}$. Error bars are defined as one standard error of the fitting parameters in the Lorentzian fit to the Raman modes.

Having described the Raman spectra evolution with increasing magnetic field, we show in Fig. 1(c) both Raman spectra in the LL and LR channels at our highest available magnetic field of $7 \mathrm{~T}$. Comparing to the spectra taken at $0 \mathrm{~T}$ in Fig. 1(a), one of the $M_{0}$ modes shifts up to nearly $9 \mathrm{~cm}^{-1}$ with its spectral intensity primarily in the LR channel, while neither $M_{1}$ nor $M_{2}$ are present in either the LR or LL channel. In addition, observable fractions of phonon intensities leak into the corresponding orthogonal channels, suggesting that the crystalline symmetry is lowered from the $C_{3 i}$ point group at $0 \mathrm{~T}$. So far, we establish that both the magnetic order and the crystalline structure change across a critical magnetic field $B_{c}$ of about $2 \mathrm{~T}$. To gain more insight into these magnetic-field-induced phase transitions and their relationships to one the other, in the following, we first discuss the magnetic phase transition from the magnetic field dependence of $M_{0}$ and then address the structural phase transition from the combination of the magnetic field dependence of the $M_{1-2}, A_{g}$, and $E_{g}$ phonons.

\section{B. Zeeman shift of spin waves}

To provide a comprehensive picture of the magnetic field dependence of $M_{0}$, we summarize in Fig. 2(a) the key experimental result of the $M_{0}$ frequencies shifting as a function of the external magnetic field and the corresponding field-dependent spin-wave calculations in the left and right panels, respectively. Here, we show the average $M_{0}$-type mode frequencies from the Lorentzian fit of the Raman spectra for both the Stokes and anti-Stokes shift in both the LL and LR channels. Strikingly, despite the fact that bulk $\mathrm{CrI}_{3}$ is considered as a simple Ising ferromagnet [13], we observe that three spin-wave branches at magnetic fields lower than $2 \mathrm{~T}$ collapse into one across $B_{c}=2 \mathrm{~T}$. In particular, a pair of the three branches below $2 \mathrm{~T}$ start with close frequencies of approximately 3.4 and $3.9 \mathrm{~cm}^{-1}$ at $0 \mathrm{~T}$ and evolve in opposite trends at increasing magnetic field $\left(M_{0 a}\right.$ and $\left.M_{0 b}\right)$, while the third one increases linearly since its appearance at approximately $1 \mathrm{~T}$ and continues after $B_{c}$ of $2 \mathrm{~T}$ with a weak discontinuity of frequency redshift $\left(M_{0 c}\right)$. The field dependence of $M_{0 a}$ and $M_{0 b}$ is typical behavior of spin waves in AFMs in which the angular momenta of two degenerate spin waves align parallel and antiparallel, respectively, to the external magnetic field, and $M_{0 c}$ is consistent with spin waves in FMs.

Our experimental observations above suggest a mixed state of layered AFM and $\mathrm{FM}$ for a $3 \mathrm{D} \mathrm{CrI}_{3}$ bulk, in contrast to the literature assignment of a pure FM 
(a)

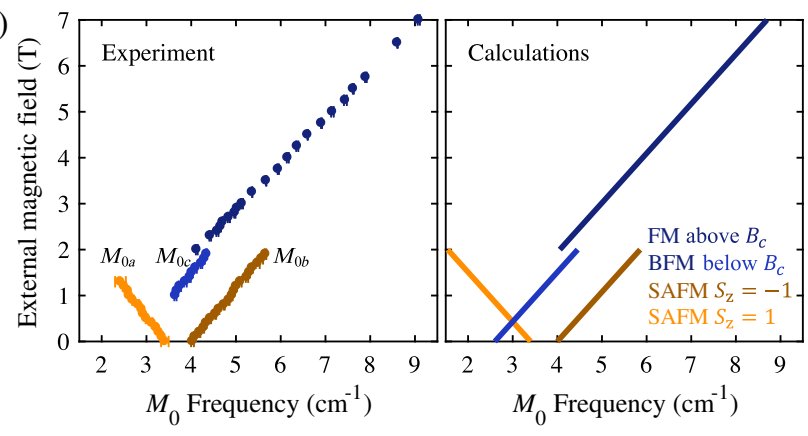

(b)

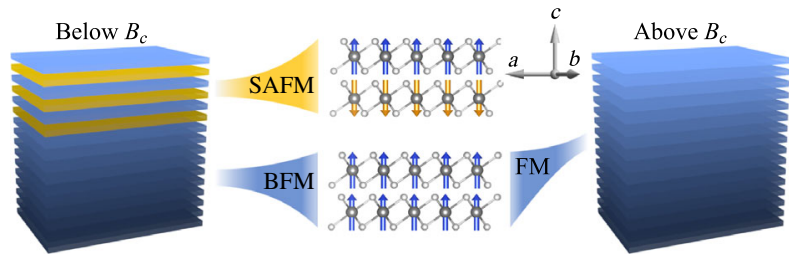

FIG. 2. Establishing the mixed SAFM and BFM phase in 3D $\mathrm{CrI}_{3}$. (a) Experimental data (left) and spin-wave calculations (right) for the magnetic field dependence of $M_{0}$-type mode frequencies. $M_{0 a}, M_{0 b}$, and $M_{0 c}$ label the three spin-wave branches below the critical magnetic field $B_{c}$ of $2 \mathrm{~T}$. Error bars correspond to one standard error of the fitted central frequencies for the $M_{0}$-type Raman modes and are mostly smaller than the data point symbols. (b) Schematic illustration of the mixed state of the surface-layered AFM (SAFM for the state with alternating spin moments in the adjacent layers) and the deep bulk FM (BFM for the state with all the spin moments along one direction) below $B_{c}$ and the pure FM state above $B_{c}$.

phase [13]. This suggestion is consistent with the absence of $M_{0 c}$ below $B_{c}$ in $\mathrm{CrI}_{3}$ flakes (see Supplemental Material Sec. 3 [23]). As sketched in Fig. 2(b), below $B_{c}$, top layers of bulk $\mathrm{CrI}_{3}$ show layered AFM (denoted as SAFM) that is similar to what has been reported in $2 \mathrm{D} \mathrm{CrI}_{3}$ thin films [3-6], and deeper bulk exhibits a FM order (denoted as BFM) that is consistent with what bulk magnetization measurements find [30]. The small energy scale of about $1 \mu \mathrm{eV}$ [31] of the long-range magnetic dipole-dipole interactions that favor the interlayer FM is much weaker than the interlayer AFM exchange coupling of $150 \mu \mathrm{eV}$. Therefore, we rule out the possibility of pure magnetic energetic reasons and require surface reconstructions to establish the interlayer AFM coupling at the surface while having FM coupling in the bulk. Hence, the SAFM provides the pair of spin waves with opposite Zeeman shifts $\left(M_{0 a}\right.$ and $\left.M_{0 b}\right)$ while the BFM leads to the third branch with a linearly increasing frequency with growing magnetic field $\left(M_{0 c}\right)$. Also noted is that the BFM provides an effective magnetic field of $0.27 \mathrm{~T}$ to lift the degeneracy of two spin waves of the SAFM at the 0-T external magnetic field. Above $B_{c}$, SAFM transits into FM in the same manner as layered $\mathrm{AFM}$ in $\mathrm{CrI}_{3}$ thin flakes [3-6], leading the entire $\mathrm{CrI}_{3}$ crystal into a FM state as shown in Fig. 2(b) and resulting in a sole spin-wave branch above $B_{c}$.
This SAFM-to-FM transition in $3 \mathrm{D} \mathrm{CrI}_{3}$ bulk is further supported by a weak anomaly at $B_{c}= \pm 2 \mathrm{~T}$ in the magnetization vs magnetic field measurements (see Supplemental Material Sec. 4 [23]). Based on this proposed model, the calculated magnetic field dependence of the $M_{0}$-type mode frequencies [right, Fig. 2(a)] with a nearestneighbor Ising spin Hamiltonian coincides well with our experimental data [left, Fig. 2(a)] (see the calculation details in Supplemental Material Sec. 5 [23]). Based on the selection rule for $M_{0}$ evolving from the nearly equal weighted presence in both LL and LR channels at $0 \mathrm{~T}$ to a dominant selection in the LR channel at $7 \mathrm{~T}$, we can further infer that the spin moments in $\mathrm{CrI}_{3}$ orient from being tilted away to nearly aligned with the surface normal as the external magnetic field increases.

\section{Structural phase transition}

Concurrent with the magnetic phase transition at $B_{c}$, we also observe an evident first-order structural phase transition manifested by abrupt changes in phonons [Figs. 3(a)-3(d)]. First and foremost, the appearance of fully symmetric $A_{g}$ phonons in the LR channel [Fig. 3(a)] reveals the transformation of their Raman tensors from the $\left(\begin{array}{c}a \cdot \cdot \\ \cdot a\end{array}\right)$ to the $\left(\begin{array}{l}a \\ \cdot b\end{array}\right)$ form, suggesting the loss of the threefold rotational symmetry and thus the breaking of the rhombohedral crystal symmetry. We propose the shearing of VDW layers away from the aligned ABC stacking order [Fig. 3(e)], which is indeed a structural instability for $\mathrm{CrI}_{3}$ bulk $[13,32]$, as one means to transit from the $C_{3 i}$ rhombohedral (nearly $D_{3 d}$ because of weak interlayer interactions) to the $C_{2 h}$ monoclinic crystal symmetry (see Supplemental Material Sec. 1 [23] for $C_{2 h}$ Raman selection rules and Sec. 6 for its comparison to the high-temperature monoclinic structure). Interestingly, this magnetic-field-induced monoclinic phase mimics a 3D quantum nematic long-range order with a director as its order parameter which breaks the rotational symmetry but preserves the lattice translational symmetry [indicated as elongated ellipses in Fig. 3(e)]. It is known that such a 3D quantum nematic order must emerge through the first-order phase transition [33], as is indeed our case here. Second, for the $E_{g}$ phonons, which are present only in the LR channel below $B_{c}$, some of their intensities exhibit leakage into the LL channel right across $B_{c}$ while some others show clear jumps in the LR channel at $B_{c}$ without leaking into the LL channel [examples of each case are shown in Figs. 3(b) and 3(c)]. These two cases are consistent with the $E_{g}\left(C_{3 i}\right)$ phonons transforming into the $A_{g}\left(C_{2 h}\right)$ and $B_{g}\left(C_{2 h}\right)$ phonons, respectively, which further corroborates the proposed structural phase transition at $B_{c}$. Third, the antisymmetric $M_{1,2}$ modes stay nearly constant until $B_{c}$ and disappear right above $B_{c}$ [see $M_{2}$ in Fig. 3(d) and $M_{1}$ in Supplemental Material Sec. 7 [23]]. Both modes were initially interpreted as surface magnetic excitations based 
(a)

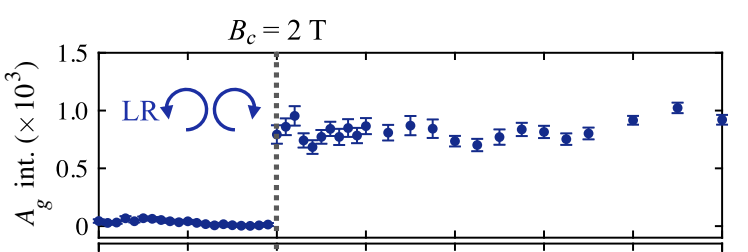

(b)

(c)

(d)

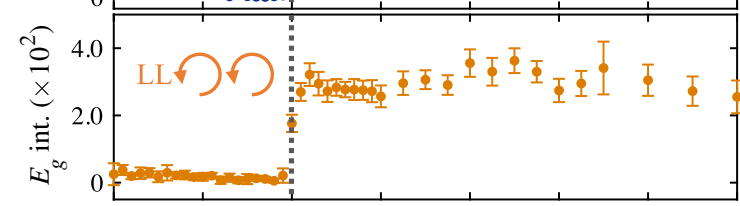

c)
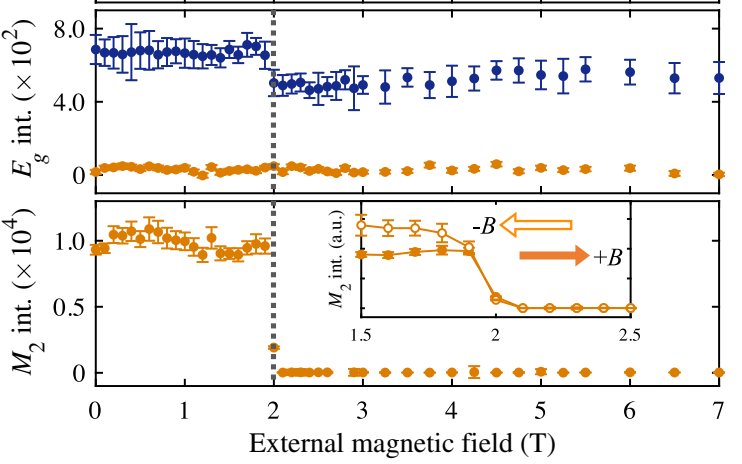

(e)

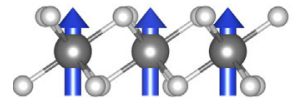

SAFM

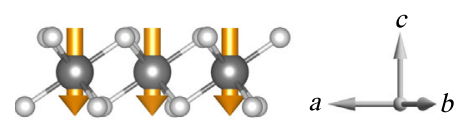

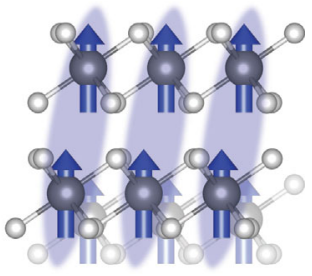

FM
FIG. 3. Revealing the rhombohedral-to-monoclinic structural phase transition at $B_{c}$ in $3 \mathrm{D} \mathrm{CrI}_{3}$. Magnetic field dependence of (a) a representative $A_{g}$ phonon (at approximately $129 \mathrm{~cm}^{-1}$ ) intensity leakage into the LR channel, (b) an example of an $E_{g}$ phonon (at approximately $109 \mathrm{~cm}^{-1}$ ) intensity showing up in the LL channel, which corresponds to the $E_{g}$ phonon of the $C_{3 i}$ structure [i.e., $E_{g}\left(C_{3 i}\right)$ ] transforming into the $A_{g}$ phonon of the $C_{2 h}$ structure [i.e., $A_{g}\left(C_{2 h}\right)$ ], (c) an example of the $E_{g}$ phonon (at approximately $240 \mathrm{~cm}^{-1}$ ) intensity experiencing a clear discontinuity in the LR channel but remaining absent in the LL channel corresponding to an $E_{g}\left(C_{3 i}\right)$ phonon transforming into a $B_{g}\left(C_{2 h}\right)$ mode, and (d) $M_{2}$-mode intensity. Inset shows an enlargement between 1.5 and 2.5 T with both increasing $(+B)$ and decreasing $(-B)$ magnetic fields. Error bars correspond to one standard error of the fitted peak intensities. (e) Schematic illustration of the shearing of $\mathrm{CrI}_{3}$ layers across the magnetic phase transition $B_{c}$. The light blue ellipses represent the directors between layers formed because of this lattice deformation.

on their broken time-reversal symmetry and thickness independence [15]. Here, we can immediately rule out the possibility that they are conventional bulk spin waves based on the magnetic field independence of their frequencies below $B_{c}$, and we can confidently associate them with the SAFM because of their disappearance above $B_{c}$. Based on this finding, we propose one possible origin of $M_{1,2}$ to be a collective excitation made of two parts, one being the $c$-axis zone boundary phonon of the nonmagnetic lattice $A\left(\vec{k}_{c}, \omega\right)$ and the other being the layered AFM order $M\left(-\vec{k}_{c}, 0\right)$, where $\vec{k}_{c}$ is the out-of-plane wave vector for the constituent phonon $(A)$ and magnetism $(M)$ at frequencies of $\omega$ and 0 (i.e., elastic scattering off the layered AFM order), respectively. Such a collective excitation breaks the time-reversal symmetry, possesses a total momentum of zero, and becomes inaccessible in the FM phase above $B_{c}$ due to the finite momenta (see detailed analysis in Supplemental Material Sec. 8 [23]). In other words, $M_{1,2}$ corresponds to a special zone folding by a single copy (or more precisely, an odd number of copies) of the magnetic order that breaks the time-reversal symmetry. However, this proposed origin cannot account for the thickness independence reported in Ref. [15]. Further experiments are needed to pin down the exact nature of both $M_{1}$ and $M_{2}$ modes. Nevertheless, they are good indicators for the SAFM state and the rhombohedral crystal lattice [Figs. 3(d) and 1(d)]. In contrast to conventional structural transitions, the emergence of this 3D quantum nematic order is driven by an external magnetic field that has a much stronger coupling to electrons than to ions, supporting an electronic origin for this structural transition. One natural mechanism for it could be that this interlayer shear deformation increases the distance between the nearest interlayer spins and thereby reduces the exchange energy penalty for the field-induced layered AFM-to-FM transition. Magnetic field-dependent Raman data on $\mathrm{CrI}_{3}$ flakes show consistent results (see Supplemental Material Sec. 9 [23]).

\section{Temperature vs magnetic field phase diagram}

We now proceed to construct the temperature versus magnetic field phase diagram for bulk $\mathrm{CrI}_{3}$ by performing temperature- (magnetic field-) dependent Raman measurements at multiple magnetic fields (temperatures). We show in Fig. 4(a) the temperature dependence of the $M_{0 c}$ FM frequency at a series of magnetic fields, and we clearly observe a crossover between FM and paramagnetic (PM) states at approximately $65 \mathrm{~K}$ at all applied magnetic fields $[33,34]$. This crossover is represented by the striped line with the experimental data points from this work in Fig. 4(c), and its extrapolation to zero magnetic field indeed corroborates the FM transition temperature of $61 \mathrm{~K}$ obtained from the magnetization measurements in $3 \mathrm{D} \mathrm{CrI}_{3}$ bulk [13]. Meanwhile, we display in Fig. 4(b) the magnetic field dependence of the $M_{2}$ intensity at several temperatures, and we discover a decreasing trend of the critical magnetic field $B_{c}$ with increasing temperatures. This observation shows that the novel mixed state of SAFM and $\mathrm{BFM}$ in bulk $\mathrm{CrI}_{3}$ is bounded by a line of phase transitions of both the layered AFM-to-FM and the rhombohedral-to-monoclinic structures, which is highlighted by a solid brown line in Fig. 4(c). Furthermore, 

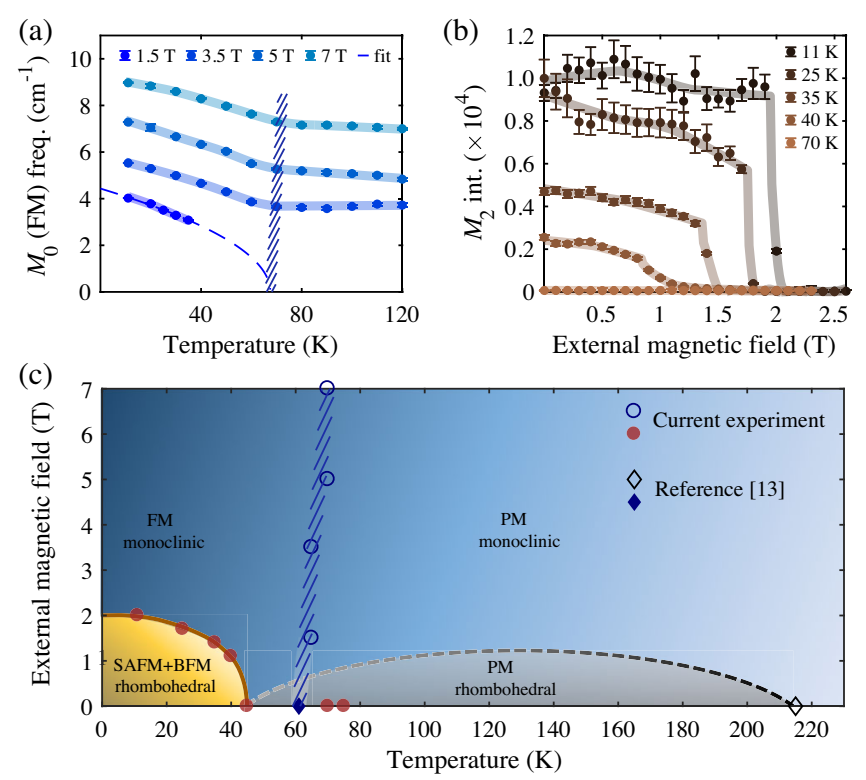

FIG. 4. Constructing the temperature vs external magnetic field phase diagram for $3 \mathrm{D} \mathrm{CrI}_{3}$. (a) Temperature dependence of the $M_{0 c}$ frequency for the FM spin-wave branch at multiple magnetic fields of $1.5 \mathrm{~T}$ (below $B_{c}$ ), 3.5, 5, and $7 \mathrm{~T}$ (above $B_{c}$ ). The dashed line is a fit to the data taken at $1.5 \mathrm{~T}$, while the solid lines are guide to the eyes. The striped pattern highlights the crossover between the paramagnetism (PM) and ferromagnetism (FM). (b) Magnetic field dependence of the $M_{2}$ intensity at multiple temperatures of $11,25,35,40 \mathrm{~K}$ (below $T_{c}$ ), and $70 \mathrm{~K}$ (above $T_{c}$ ). Error bars correspond to one standard error of the fitted parameters. (c) A temperature vs external magnetic phase diagram constructed based on both the findings from the current work (blue open and brown filled circles) and the literature (blue open and filled diamonds, Ref. [13]). The brown solid line represents a true phase boundary between the mixed magnetic phase with a rhombohedral structure and the FM phase with a monoclinic structure. The gray dashed line is for a potential phase boundary between the rhombohedral and monoclinic PM phases. The blue stripped pattern indicates the crossover between the PM and FM phases.

based on the fact that the crystal structure of $3 \mathrm{D} \mathrm{CrI}_{3}$ bulk transits from the rhombohedral to monoclinic symmetry across approximately $220 \mathrm{~K}$ from below at $0 \mathrm{~T}$ [13], we propose the presence of a phase boundary for the paramagnetic rhombohedral phase so as to connect the two regions of monoclinic structures above $220 \mathrm{~K}$ at $B=0 \mathrm{~T}$ and below $45 \mathrm{~K}$ at $B>B_{c}$. Please note that while the starting point of this phase boundary is determined to be at $T=220 \mathrm{~K}, B=0 \mathrm{~T}$, its end point is unknown due to a lack of experimental data, and thus, the gray dashed line in Fig. 4(c) presents only one possibility.

\section{SUMMARY AND CONCLUSION}

Our findings in 3D $\mathrm{CrI}_{3}$ bulk of a novel mixed state of the surface-layered AFM and the bulk FM and a magnetic-field-induced first-order structural phase transition reveal a rich phase diagram for this VDW magnetic semiconductor, which unambiguously resolves the puzzling evolution from the FM in $3 \mathrm{D}$ to the layered AFM in $2 \mathrm{D} \mathrm{CrI}_{3}$. Furthermore, controlling this unique magnetism in VDW magnets and its interplay with crystalline structures opens up new possibilities for the realization of novel 2D magnetic phases and the applications in modern spintronics. While a broader class of VDW magnets with such a unique magnetism and strong magnetoelastic coupling are to be identified, $\mathrm{CrI}_{3}$ serves as an ideal platform to explore a variety of external controls, such as electric field, strain, and charge-carrier doping, on the magnetism and its interplay with other degrees of freedoms.

\section{METHODS}

\section{A. Growth of $\mathrm{CrI}_{3}$ single crystals}

Single crystals of $\mathrm{CrI}_{3}$ are grown by the chemical-vaportransport method. Chromium power (99.99\% purity) and iodine flakes $(99.999 \%)$ in a 1:3 molar ratio are put into a silicon tube with a length of $200 \mathrm{~mm}$ and an inner diameter of $14 \mathrm{~mm}$. The tube is pumped down to $0.01 \mathrm{~Pa}$ and sealed under vacuum and then placed in a two-zone horizontal tube furnace. The two growth zones are raised slowly to 903 and $823 \mathrm{~K}$ for two days and are then held there for another seven days. Shiny, black, platelike crystals with lateral dimensions of up to several millimeters can be obtained from the growth. In order to avoid degradation, the $\mathrm{CrI}_{3}$ crystals are stored in a nitrogen-filled glovebox and exfoliated in the dark to expose fresh surfaces right before the experiment (the air exposure time in a dark environment was less than $10 \mathrm{~s}$ before the sample is sealed in the cryostat vacuum chamber).

\section{B. Micro-Raman spectroscopy}

Micro-Raman spectroscopy measurements are carried out using a 632.81-nm excitation laser with a full width at half maximum of $0.85 \mathrm{~cm}^{-1}$ on resonance with the charge transfer and $\mathrm{Cr}^{3+}$ to ${ }^{4} A_{1}$ transitions of $\mathrm{CrI}_{3}$ in order to increase the Raman sensitivity. According to the absorption measurements taken in $\mathrm{CrI}_{3}$ flakes, the optical penetration depth in $\mathrm{CrI}_{3}$ for $633 \mathrm{~nm}$ is on the order of 30 layers. The laser beam on the sample site is focused down to approximately $3 \mu \mathrm{m}$ in diameter, and the laser power is kept at $80 \mu \mathrm{W}$, which corresponds to a similar fluence used in the literature $(10 \mu \mathrm{W}$ over a $1-\mu$ m-diameter area) to minimize the local heating effect. Backscattering geometry is used. The scattered light is dispersed by a Horiba LabRAM HR Evolution Raman microscope (1800 groves/mm grating) from Horiba Scientific and detected by a thermoelectric cooled CCD camera provided by Horiba Scientific as well. Because of the small sample size on the order of a few microns, we use the confocal microscope geometry at a magnification of $40 \times$ to position the laser spot onto the sample of interest and perform a Raman spectroscopy experiment. In order 
TABLE I. Transmission efficiency and their corresponding Stokes and anti-Stokes edges for the notch filter used in the experiment with a 632.81-nm excitation laser. The transmission efficiency is less than $10 \%$ in $[-1.8,3.7] \mathrm{cm}^{-1}$, between $10 \%$ and $50 \%$ over the ranges of $[3.7,4.2]$ and $[-2.2,-1.8] \mathrm{cm}^{-1}$, and between $50 \%$ and $90 \%$ over the ranges of $[4.2,7.8]$ and $[-6.3,-2.2] \mathrm{cm}^{-1}$.

\begin{tabular}{lcc}
\hline \hline $\begin{array}{l}\text { Transmission } \\
\text { efficiency }\end{array}$ & $\begin{array}{c}\text { Stokes edge } \\
\left(\mathrm{cm}^{-1}\right)\end{array}$ & $\begin{array}{c}\text { Anti-Stokes edge } \\
\left(\mathrm{cm}^{-1}\right)\end{array}$ \\
\hline $10 \%$ & 3.7 & -1.8 \\
$50 \%$ & 4.2 & -2.2 \\
$90 \%$ & 7.8 & -6.3 \\
\hline \hline
\end{tabular}

to suppress the reflected light and Rayleigh scattering, we add a notch filter with a central wavelength of $632.85 \mathrm{~nm}$, with its transmission profile described in Table I. A commercial variable temperature $(<10-325 \mathrm{~K})$, closedcycle, microscopy cryostat from Cryo Industries of America, Inc. is interfaced with the Raman microscope. A commercial cryogen-free room-temperature-bore ( 2 in diameter and 6.88 in long) superconducting magnet from Cryo Industries of America, Inc. is used to achieve the variable out-of-plane magnetic field from 0 to $7 \mathrm{~T}$. The cryostat cold finger, on which the samples are mounted, is inserted into the center of the room-temperature bore of the magnet. In this work, the selection rule measurements are performed mainly under the circularly polarized basis to eliminate any artifacts of the Faraday effect from the microscope objective subject to the strong stray magnetic field. All thermal cycles are performed at a base pressure lower than $7 \times 10^{-7}$ torr.

\section{ACKNOWLEDGMENTS}

We acknowledge helpful discussions with Xiaodong $\mathrm{Xu}$, Roberto Merlin, and Elizabeth Drueke. L. Z. acknowledges support by NSF CAREER Grant No. DMR1749774. R. H. acknowledges support by NSF CAREER Grant No. DMR-1760668 and NSF MRI Grant No. DMR1337207. K. S. acknowledges support through NSF Grant No. NSF-EFMA-1741618. A. W. T. acknowledges support from the U.S. Army Research Office (Grant No. W911NF19-10267) and an Ontario Early Researcher Award (ER1713-199). This research was undertaken thanks in part to funding from the Canada First Research Excellence Fund. H. L. acknowledges support by the National Key R\&D Program of China (Grant No. 2016YFA0300504), the National Natural Science Foundation of China (Grants No. 11574394, No. 11774423, and No. 11822412), the Fundamental Research Funds for the Central Universities, and the Research Funds of Renmin University of China (Grants No. 15XNLQ07, No. 18XNLG14, and No. 19XNLG17).

The authors declare no competing financial interests.
S. L., Z. Y., and X. L. contributed equally to this work. L.Z. and R. H. conceived and initiated this project; S. T., C. L., and H.L. synthesized and characterized the bulk $\mathrm{CrI}_{3}$ single crystals; Z. Y., G. Y., and R. H. performed the Raman measurements; S.L. performed the magnetic field-dependent spin-wave dispersion calculations under the guidance of K.S. and L.Z.; H.H.K., B.Y., and A.W.T. made the $\mathrm{CrI}_{3}$ flake samples and performed magnetotunneling resistivity measurements. S. L., X. L., and L. Z. analyzed the data; S. L., X. L., R. H., and L. Z. wrote the manuscript, and all authors participated in the discussions of the results.

[1] C. Gong et al., Discovery of Intrinsic Ferromagnetism in Two-Dimensional van der Waals Crystals, Nature (London) 546, 265 (2017).

[2] B. Huang et al., Layer-Dependent Ferromagnetism in a van der Waals Crystal down to the Monolayer Limit, Nature (London) 546, 270 (2017).

[3] D. R. Klein et al., Probing Magnetism in 2D van der Waals Crystalline Insulators via Electron Tunneling, Science 360, 1218 (2018).

[4] T. Song et al., Giant Tunneling Magnetoresistance in SpinFilter van der Waals Heterostructures, Science 360, 1214 (2018).

[5] H. H. Kim, B. Yang, T. Patel, F. Sfigakis, C. Li, S. Tian, H. Lei, and A. W. Tsen, One Million Percent Tunnel Magnetoresistance in a Magnetic van der Waals Heterostructure, Nano Lett. 18, 4885 (2018).

[6] Z. Wang, I. Gutiérrez-Lezama, N. Ubrig, M. Kroner, M. Gibertini, T. Taniguchi, K. Watanabe, A. Imamoğlu, E. Giannini, and A.F. Morpurgo, Very Large Tunneling Magnetoresistance in Layered Magnetic Semiconductor $\mathrm{CrI}_{3}$, Nat. Commun. 9, 2516 (2018).

[7] J.-U. Lee, S. Lee, J. H. Ryoo, S. Kang, T. Y. Kim, P. Kim, C.-H. Park, J.-G. Park, and H. Cheong, Ising-Type Magnetic Ordering in Atomically Thin $\mathrm{FePS}_{3}$, Nano Lett. 16, 7433 (2016).

[8] K. Kim et al., Antiferromagnetic Ordering in van der Waals 2 D Magnetic Material $\mathrm{MnPS}_{3}$ Probed by Raman Spectroscopy, 2D Mater. 6, 041001 (2019).

[9] K. Kim, S. Y. Lim, J.-U. Lee, S. Lee, T. Y. Kim, K. Park, G. S. Jeon, C.-H. Park, J.-G. Park, and H. Cheong, Suppression of Magnetic Ordering in XXZ-Type Antiferromagnetic Monolayer $\mathrm{NiPS}_{3}$, Nat. Commun. 10, 345 (2019).

[10] S. Jiang, J. Shan, and K. F. Mak, Electric-Field Switching of Two-Dimensional van der Waals Magnets, Nat. Mater. 17, 406 (2018).

[11] B. Huang et al., Electrical Control of 2D Magnetism in Bilayer $\mathrm{CrI}_{3}$, Nat. Nanotechnol. 13, 544 (2018).

[12] S. Jiang, L. Li, Z. Wang, K. F. Mak, and J. Shan, Controlling Magnetism in $2 \mathrm{D} \mathrm{CrI}_{3}$ by Electrostatic Doping, Nat. Nanotechnol. 13, 549 (2018).

[13] M. A. McGuire, H. Dixit, V. R. Cooper, and B. C. Sales, Coupling of Crystal Structure and Magnetism in the Layered, Ferromagnetic Insulator $\mathrm{CrI}_{3}$, Chem. Mater. 27, 612 (2015). 
[14] L. Chen, J. H. Chung, B. Gao, T. Chen, M. B. Stone, A. I. Kolesnikov, Q. Huang, and P. Dai, Topological Spin Excitations in Honeycomb Ferromagnet $\mathrm{CrI}_{3}$, Phys. Rev. X 8, 041028 (2018).

[15] W. Jin et al., Raman Fingerprint of Two Terahertz Spin Wave Branches in a Two-Dimensional Honeycomb Ising Ferromagnet, Nat. Commun. 9, 5122 (2018).

[16] K. L. Seyler et al., Ligand-Field Helical Luminescence in a 2D Ferromagnetic Insulator, Nat. Phys. 14, 277 (2018).

[17] P. A. Fleury and R. Loudon, Scattering of Light by One- and Two-Magnon Excitations, Phys. Rev. 166, 514 (1968).

[18] W. Hayes and R. Loudon, Scattering of Light by Crystals (Dover Publication, New York, 2012).

[19] B. Huang, J. Cenker, X. Zhang, E. L. Ray, T. Song, T. Taniguchi, K. Watanabe, M. A. McGuire, D. Xiao, and X. $\mathrm{Xu}$, Tuning Inelastic Light Scattering via Symmetry Control in the Two-Dimensional Magnet $\mathrm{CrI}_{3}$, Nat. Nanotechnol., 15, 212 (2020).

[20] A. McCreary et al., Distinct Magneto-Raman Signatures of Spin-Flip Phase Transitions in $\mathrm{CrI}_{3}$, arXiv:1910.01237.

[21] Y. Zhang et al., Magnetic Order-Induced Polarization Anomaly of Raman Scattering in $2 \mathrm{D}$ Magnet $\mathrm{CrI}_{3}$, Nano Lett. 20, 729 (2020).

[22] J. Ji, A. Zhang, J. Fan, Y. Li, X. Wang, J. Zhang, E. W. Plummer, and Q. Zhang, Giant Magneto-Optical Raman Effect in a Layered Transition Metal Compound, Proc. Natl. Acad. Sci. U.S.A. 113, 2349 (2016).

[23] See Supplemental Material at http://link.aps.org/ supplemental/10.1103/PhysRevX.10.011075 for additional information for Raman selection rules in the circular polarization basis, magnetic field-dependent Raman spectra taken in different polarization channels and on samples of different thicknesses, magnetization measurement results, and spin wave calculations.

[24] D. Shcherbakov et al., Raman Spectroscopy, Photocatalytic Degradation, and Stabilization of Atomically Thin Chromium Tri-Iodide, Nano Lett. 18, 4214 (2018).

[25] S. Djurdjić-Mijin, A. Solajic, J. Pesic, M. Scepanovic, Y. Liu, A. Baum, C. Petrovic, N. Lazarevic, and Z. V. Popovic, Lattice Dynamics and Phase Transition in $\mathrm{CrI}_{3}$ Single Crystals, Phys. Rev. B 98, 104307 (2018).

[26] L. Webster, L. Liang, and J.-A. Yan, Distinct Spin-Lattice and Spin-Phonon Interactions in Monolayer Magnetic $\mathrm{CrI}_{3}$, Phys. Chem. Chem. Phys. 20, 23546 (2018).

[27] D. T. Larson and E. Kaxiras, Raman Spectrum of $\mathrm{CrI}_{3}$ : An Ab Initio Study, Phys. Rev. B 98, 085406 (2018).

[28] N. W. Ashcroft and N. D. Mermin, Solid State Physics (Holt, Rinehart and Winston, New York, 1976).

[29] J. C. Tolédano and P. Tolédano, in Magnetic and Liquid Crystal Systems (World Scientific, Singapore 1987).

[30] B. Niu et al., Coexistence of Magnetic Orders in Two-Dimensional Magnet $\mathrm{CrI}_{3}$, Nano Lett. 20, 553 (2020).

[31] D. C. Johnston, Magnetic Dipole Interactions in Crystals, Phys. Rev. B 93, 014421 (2016).

[32] N. Sivadas, S. Okamoto, X. Xu, C. J. Fennie, and D. Xiao, Stacking-Dependent Magnetism in Bilayer $\mathrm{CrI}_{3}$, Nano Lett. 18, 7658 (2018).

[33] P. M. Chaikin and T. C. Lubensky, Principles of Condensed Matter Physics (Cambridge University Press, Cambridge, England, 2000).

[34] J. Cardy, P. Goddard, and J. Yeomans, Scaling and Renormalization in Statistical Physics (Cambridge University Press, Cambridge, England, 1996). 